\title{
An Universal Interconnection System to Connect Distributed Generation to the Grid
}

\author{
Vinod John, Member IEEE, Eric Benedict, Member IEEE, and Shazreen Meor Danial
}

\begin{abstract}
Interconnection equipment between distributed energy resources (DER) and the grid is typically custom designed by the distributed generation (DG) equipment manufacturer or integrated by engineering firms using subcomponents such as relays, sensors and switchgear. The DER Switch described in the paper has integrated all of the required equipment for the DER interconnection into a single package that is designed to be compliant with IEEE 1547 and UL 1741 standards. A 480V, 200A, circuit breaker based DER Switch prototype with a digital signal processing (DSP) board was designed, built and tested. The objective was to create a standard, flexible universal interface switch for distributed energy resources so that single or multiple DER systems like wind turbines or solar arrays can be connected to a utility. The resulting interconnection switch design is DER technology neutral and can be used for inverter and machine DG applications.
\end{abstract}

Index Terms-- Distributed generation, universal interconnection, synchronization, islanding, protective relaying.

\section{INTRODUCTION}

$\mathrm{I}_{\mathrm{N}}^{\mathrm{N}}$ NTEGRATION of distributed energy resources (DER) with the electric power system is increasingly seen as a technology that can change the traditional method of electrical power delivery and can provide multiple advantages to energy customers, energy suppliers, and society overall [1]. Numerous promising generation, storage, and load management technologies are under development or are entering early commercialization stages. It is becoming increasingly apparent that new systems level technology and functionality are necessary to unlock the full potential of the emerging DER technology and to ensure a broad acceptance of DER systems as a key component in the overall energy delivery system. This paper describes an universal grid interconnection prototype that integrates multiple functions of switching, sensing, control,

This work was supported by the National Renewable Energy Laboratory (NREL) and California Energy Commission (CEC) under NREL Subcontract ZAT-4-32616-05.

Vinod John is with the Department of Electrical Engineering, Indian Institute of Science, Bangalore 560032, Karnataka, India, (e-mail: vjohn@ee.iisc.ernet.in).

Eric Benedict and Shazreen Meor Danial are with Northern Power Systems, Waitsfield Vermont 05602, USA, (e-mail: ebenedict@northernpower.com and smeor@northernpower.com).

\section{0-7803-9772-X/06/\$20.00 C2006 IEEE}

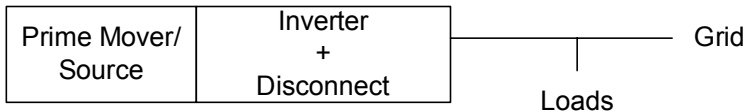

(a)

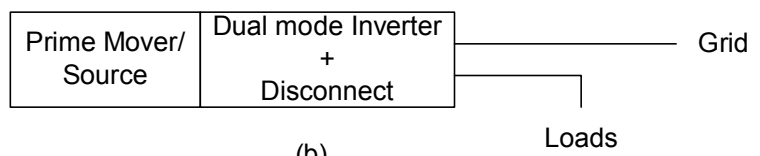

(b)

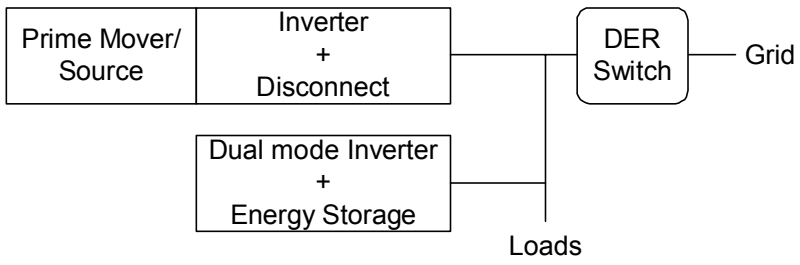

(c)

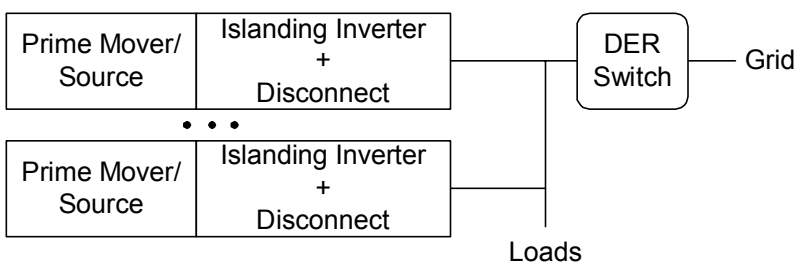

(d)

Fig. 1. DG architecture showing (a) Grid parallel configuration. (b) Dual mode inverter with grid parallel and stand alone capability and do not require additional DER Switch. (c) Upgrade of grid parallel configuration to provide standalone backup capability. (d) Multiple DG configuration that requires additional DER switch.

protection and communications into a single package.

\section{TECHNICAL APPROACH}

The approach taken to create the universal interconnection system was to define the system architecture, and to create a DER switch specification and perform design and component selection for prototype fabrication. It was seen that existing switch configurations offer basic solutions for DER interconnections. The DER Switch design addresses some of the issues and concerns for the interconnection especially related to intentional islanding. In addition, a DER Switch can be used to improve the services provided by the DER in terms of load power quality [2]-[4]. These improvements are provided through the combination of the DER Switch's advanced controller and higher possible switching speeds along with DGs with appropriate control capabilities. There are many DER applications where the interconnection switch is integrated with the DER itself. This approach can be effective in applica- 
tions where a cost effective DER package can be built and shipped out to a customer for installation. This is especially true for low power single-phase applications. However different customers may need different combinations of DER equipment to meet their needs. To broaden the range of DER options there can be advantages to decouple the DG from the utility interconnection switch. Two related questions need to be considered in this context. The first is, when does a separate DER Switch make sense? And the second is, what applications require the DER Switch to be a separate entity from the DG? This requires one to look at specific DG system architectures where such a separate switch is desirable.

The DG can be operated either as a grid connected or a stand-alone configuration. A basic grid connected DG system as shown in Fig. 1(a) does not require an additional interconnection device under normal operation. If the operation of the DG system as an intentional island is required, then there are multiple architectures that can be utilized. Some inverter based DG systems are available from DG vendors with dual mode capability as shown in Fig. 1(b). These systems have some internal energy storage capacity, which is utilized to obtain an acceptable response to step load changes. These dual mode DG systems may have explicit external or internal switchgear that is used to transfer between standalone and grid parallel operation. When the external switchgear is present, the inverter typically needs to know the open/closed status of the switch on an instantaneous basis and this requires a highspeed control interface between the switch and inverter controller.

Fig. 1(c) shows a grid connected DG system that has been upgraded to operate in standalone mode by adding an inverter with intentional islanding capability and a DER Switch. This configuration can be used to upgrade a grid parallel DG configuration to one that has intentional islanding capability. This configuration can have a higher cost because it makes use of two inverters. However, a more flexible system configuration is achieved because the two inverters and the DER Switch can operate with some physical separation between them and without the necessity for high-speed control interconnections. Replacing the dual mode DG inverter controls with controls that can seamlessly operate in grid connected and islanded modes of operation will further optimize the overall system [3]. Such a configuration can be extended to the case of multiple DG systems that operate together in a Microgrid power network. This architecture that is shown in Fig. 1(d), leads to a simpler overall system design. In case of the configurations that utilized the DER Switch, the interconnection protection functions, such as specified in IEEE 1547, should reside at the DER Switch to prevent the power converter from unwanted trips in situations where intentional islanding is required. The islanding or dual mode inverters should have adequate provision to facilitate this coordination.

In general, the DER Switch lends itself to more advanced power network architectures. A representative system indicating DER assets, loads, the DER Switch and the area electric

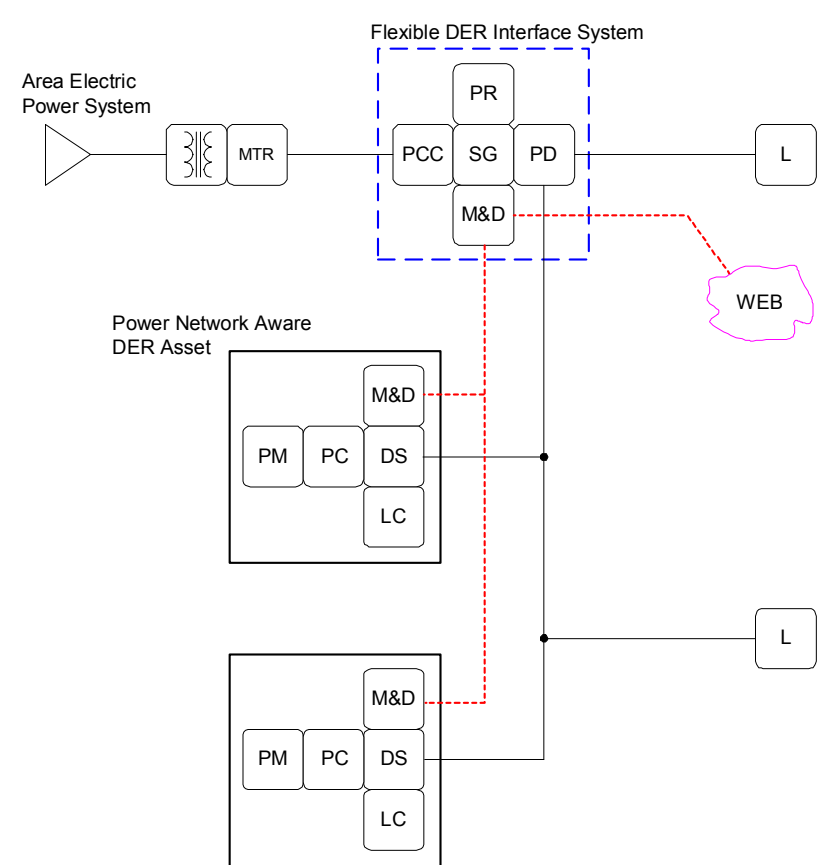

Fig. 2. DER power network block diagram indicating the target role of the DER Switch (dashed blue line envelope). MTR - meter, PCC - point of common coupling, $\mathrm{PR}$ - protective relay, $\mathrm{SG}$ - switchgear, $\mathrm{PD}$ - power distribution, M\&D - monitoring and diagnostics, PM prime mover, $\mathrm{PC}$ - power conversion, DS - DG switchgear, LC - local control, L - loads.

power system is shown in Fig. 2. The DER Switch consists of point of common coupling, switchgear, protective relay, monitoring and diagnostics and power distribution components. The communication interface can be low bandwidth between the multiple DG and the DER Switch (shown in the red dotted line) and would be used for SCADA and energy management system functions. High-speed control interconnections and communications would not be required to operate such a power network.

\section{A. Hardware Requirements}

A DER interconnection with the grid that meets the application requirements described in the previous section requires a flexible hardware concept. A traditional implementation of such a concept will involve switch hardware, voltage and current sensing devices, protective relays, a controller with diagnostic and monitoring functions, a communications processor, power supplies and other components.

The DER Switch aggregates the control functions in a digital signal processor. The hardware flexibility is retained in the DER Switch with the additional capability of replacing a circuit breaker with solid-state switches. In the case of a solidstate switch, additional breakers are used to obtain high fault interrupt rating under any internal faults and to meet Basic Impulse Insulation Level (BIL) targets when disconnected. A solid-state version of the DER Switch would be able to switch at higher speeds and provide higher power quality. The ability of the DER Switch platform to provide a range of interconnection speeds offers the flexibility to match the application requirements. The circuit breaker based DER Switch design was selected for the prototype because it was the simplest and lowest cost technology that could test all of the relay, IEEE 1547 




Fig. 3. One-line schematic of a semiconductor based DER Switch showing switch hardware, sensor locations and grid and load connections.

requirements [5] and power quality functions [2]. In the semiconductor DER Switch designs, input and output circuit breakers are used as backup protection in case the semiconductor switch fails in order to allow the DER Switch to still be able to disconnect DG from the grid. The control of the DER Switch is designed to be switch technology neutral. In addition, the same control system can be used for a DER Switch which implements the switch using either power semiconductor based components or using a circuit breaker.

The high-speed capability of the semiconductor switch and DSP allows clearing times in the range of fractions of a millisecond. This allows the capability of zero fault current contribution to the grid and the possibility of operation with network protectors that need zero reverse power flow [4]. When the DER Switch is used in combination with DER assets that can seamlessly pickup loads in cases of grid disconnection, then high power quality is available to the load. For less demanding and cost critical applications, the same design with circuit breaker switch hardware can be used.

The internal current sensors within the DER Switch are capable of measuring both ac and dc current with a high bandwidth. The external analog inputs for current measurement assume the use of 5Arms secondary CT. Analog inputs for grid and DG ac voltage measurements are made using 120 Vrms nominal secondary VT. The one-line schematic indicating analog signal sensor locations is shown in Fig. 3. Sixteen analog channels that are directly available in the DSP board for high speed sampling are utilized in the controller. Table I list the analog input and outputs used in the DER Switch controller.

Digital inputs to the DSP are optoisolated and debounced. The primary inputs from the switch controller are start, stop and reset. The application related digital inputs are interfaced with $24 \mathrm{~V}$ DC relay coils that can be externally energized. The two inputs are 1) Trip signal where a high signal indicates an external command to the DER Switch to transition to the Disconnect state, and 2) Two additional spare channels are available and can be configured either as active high or active low. Additional internal digital input signals are used to monitor the
TABLE I

ANALOG INPUT AND OUTPUT CHANNELS USED IN THE DER SWITCH DSP CONTROLLER.

\begin{tabular}{lc}
\hline \multicolumn{1}{c}{ Analog Input } & $\begin{array}{c}\text { Number of } \\
\text { channels }\end{array}$ \\
\hline Current sensor - (Is) phase A, B, C, N & $(4 \mathrm{x})$ \\
External CTs - (Ir) phase A, B, C & $(3 \mathrm{x})$ \\
Grid side sensing - (Vg) phase A, B, C & $(3 \mathrm{x})$ \\
DG side sensing - (Vdg) phase A, B, C & $(3 \mathrm{x})$ \\
Snubber circuit common and differential voltage & $(2 \mathrm{x})$ \\
Control voltage sensing (V24) & $(1 \mathrm{x})$ \\
Spare multiplexed inputs for application-related & $(2 \mathrm{x})$ \\
requirements & \\
\hline \multicolumn{1}{c}{ Analog Output } \\
\hline Spare multiplexed outputs for application-related \\
requirements
\end{tabular}

semiconductors', the circuit breakers', and the contactors' fault and status indicators.

The digital outputs of the DSP are opto-isolated. Additional interposing relays with Normal Close/Normal Open dry contacts with surge protection are used to provide isolation and voltage surge ratings. The two application-related output contacts available externally are the 'Connected or Disconnect status' of the DER Switch and an 'auxiliary switch' output. The auxiliary switch output can be used to open any circuit breaker in series with the DER Switch or trigger another DER Switch or circuit breaker to obtain a transfer switch or other more complex power switching configurations.

Optional analog zero to $5 \mathrm{~V}$ output voltage signals are available on the DSP controller board. The signals are centered at $2.5 \mathrm{~V}$ and $5 \mathrm{~V}$ represents the maximum rated output capacity. The analog output signals implemented in the DER Switch are three phase real and reactive power. Two additional spare channels can be used for the application-related signals.

\section{B. Control Requirements}

The control functions are computed based on the raw ana$\log$ and digital inputs to the DSP. A small amount of filtering is provided for EMI and noise rejection. Additional control inputs are possible through a Human Machine Interface (HMI) on a remote computer through serial communications interface. Control functions evaluate these inputs to achieve interconnection protection, to meet the IEEE 1547 standard requirements, and to evaluate ambient power quality. DG protection is left to the DG controls and is not included in the DER Switch. The DSP controller provides on-off commands for semiconductor switches and control of the circuit breakers within the DER Switch. Additional spare analog and digital outputs can be used for overall power system integration. The evaluated values of the control algorithms are available through the HMI for energy management functions.

\section{1) DER Switch State Machine}

The state machine controls the operation of the DER Switch. The primary operating states of the DER Switch are to connect the grid and DER power network or to stay disconnected. A number of the other control states are used for the startup sequence, faults and bypass operations. Fig. 4 shows the state diagram for the DER Switch controller, which is used for all the DER Switch options. It is also possible to select 


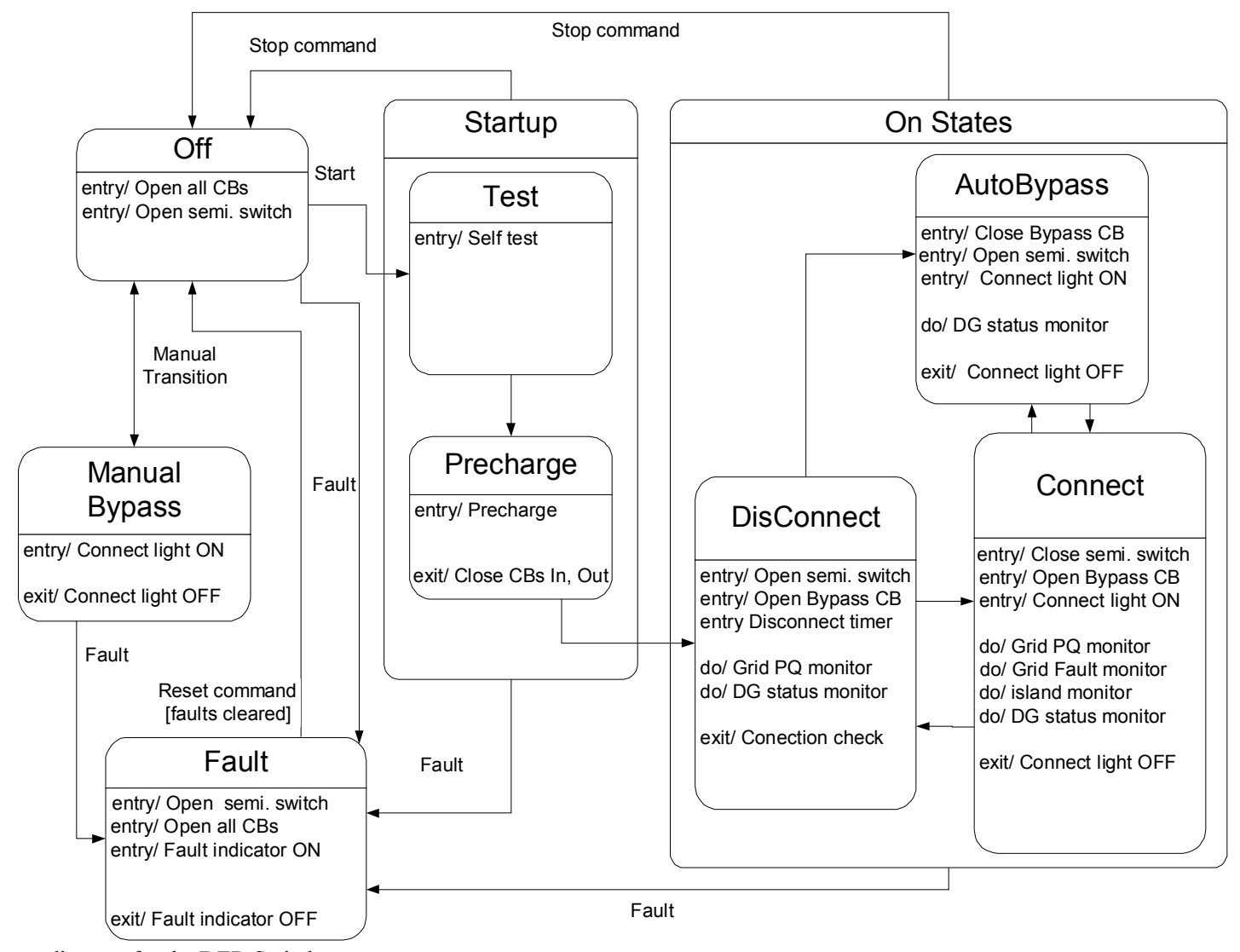

Fig. 4. Control state diagram for the DER Switch.

modes of operation of the DER Switch to obtain different behavioral characteristics within the operating states. The behavior of the DER Switch when operating in the controller states is described below.

The DER Switch following power-up and reset of the controls is considered to be the Off State. The DSP controller conditions for the Off state are: no faults are latched, all alarms are enabled, waveform capture snaplog is enabled, all semiconductors are disabled, and all discrete outputs are off. Any change in the mode settings can be done only in this state. Any CB that stays closed in this state triggers a fault. A startup sequence can be initiated from this state. The unit can transition to the Manual Bypass state if the Manual mode is set.

The condition for Manual Bypass State is similar to the Off state with the exception that the Bypass CB can be closed. A fault is triggered if the Input and Output CBs are closed in this state. There is no event-related change of state in this mode.

A start command in the Off state initiates the transition to the Test state. The motorized input and output circuit breakers should be ready to close. The grid and DER side voltages are monitored to see if they are in the nominal range and the voltage phase lock occurs on at least one side. If voltage is absent from both sides, then the unit goes to Fault, else the unit goes to the Precharge state. The Precharge state is present for semiconductor based DER Switch. The precharge contactor is closed and the voltage snubber clamp capacitor is charged up to the peak ac line to line voltage. The input and output circuit breakers are closed if the clamp voltage is above a minimum level. The precharge contactor is opened after this. A fault is generated if the clamp voltage is too low or if the input and output circuit breakers fail to close, subsequent to precharge.

In the Disconnect state the DER Switch checks to see if DER is present in the system. The DER status is obtained with a bit that is set high when DER is present or low when DER is absent. If it is present and the synchronization functions evaluate to True then the DER Switch transitions to Connect State. In the case that no DER is present, the controller checks to see if the DER side represents dead bus and the DER Switch transitions to the AutoBypass state. If there is a request to shut down the DER without de-energizing the loads through the supervisory control system, then the synchronization functions are evaluated. When the synchronization functions are true, the DER Switch transitions to the AutoBypass state. This feature is used only if there is an external supervisory controller for the DG and DER Switch system, which will ensure that the DG status is off within a short duration after reaching the AutoBypass state.

In the Connect State the DER Switch checks for power quality, anti-islanding other IEEE1547 functions and fault events. If any of these events are true, the DER Switch transitions to the Disconnect state. In case the DG is shut off (DG status is absent), the DER switch transitions to the AutoBypass state.

In the AutoBypass state the DER Switch checks to see if the DER is reconnected to the system. The DER Switch operates like a regular circuit breaker in this state and does not open in case of any power quality problems. In case DER 
status indicates that it is going to be reconnected, the DER Switch transitions to the Connect state. It is expected that the DER status will be updated before the reconnection by the Supervisory control system. In case the supervisory control is not in place, the AutoBypass state is not utilized. If the DER Switch entered the AutoBypass state because of a Request for DER shutdown, the control system should ensure that this request does not persist for a long time. The controller should time out this request and indicate a warning in case the request continues to persist.

When a fault occurs, the DER Switch can shut down rapidly into a safe state. Within this state, all CBs are open and the semiconductor switches are off. The snubber clamp circuit that is used to protect the semiconductor switch is discharged. This is the state that the DER switch enters on initial power up. The semiconductor based designs have extra input and output circuit breakers which provides backup protection in case the semiconductor-based switch fails.

In addition to the control states additional modes are provided in the controller such as: Auto/Manual mode, Local/Remote mode, Test mode and additional modes based on hardware attributes of the system.

\section{2) Control Functions}

The relay functions implemented in the DER Switch are based on typical requirements from DG projects and IEEE 1547. Additional functions are implemented for monitoring and diagnostics. Warning message and activation thresholds are provided for the control functions. Most of the relay functions trigger a transition to the Disconnect state. Exceptions are the synchronization and deadbus reclose functions that are used as enable signals for the DER Switch to reconnect. The relay functions are provided with options to enable each algorithm independently as individual functions or as a combination of functions.

The evaluation of the algorithm to check for synchronization between the grid side voltage and DG side voltage is performed in the Disconnect state. This function checks to verify that the voltage amplitude (for all three phases), frequency and phase angle are within an acceptable window to enable the closing of the DER Switch. Other DER Switch control functions are also evaluated to be true to enable the closing. Closing of the switch is only done in DER system when it is in Auto mode and if the synchronization enable is valid and reconnection enable is evaluated to be true. There are two modes for the synchronization function: 1) The absolute mode allows for synchronization when voltage, frequency and phase error magnitudes are small. 2) The Positive mode allows synchronization when frequency and phase error are small and has positive values. This is to prevent any reverse power surge during synchronization and connection from causing any trips based on fast reverse power relay calculations. The voltage magnitude and phase comparison is performed at the highest computation rate of the DSP. This ensures that any sudden jump in voltage or magnitude on either side of the DER Switch does not cause any false synchronization.

Dead bus reclosing relay function is provided in the DER Switch so that it can black start the loads connected to the
DER side of the switch, when there is no DG connected to the system. A voltage threshold and time delay is provided for coordination. This relay checks to verify that closing occurs only under a dead bus condition where the voltage is below threshold on all three phases and when DG status is OFF.

The voltages on the grid and DER side are monitored to be within acceptable ranges. A threshold and time delay is provided for coordination of protection functions. Separate voltage thresholds and time delays for the grid side and DER side are provided. An event in the Connect state will make the DSP controller transition to the Disconnected state. Any event measured on the DER side when in the Disconnect state results in an alarm to the supervisory control system.

Frequency is measured from the three phase voltage measurement on the grid and DER side. Separate frequency thresholds and time delays are provided for coordination on the grid and DER side. An event in the Connect state will make the DER Switch to transition to the Disconnected state. Any event measured on the DER side when in the Disconnect state triggers an alarm to the supervisory control system.

Additional protection functions checks for phase rotation direction, missing phase information or lack of signal on the phase voltage. In addition, this function checks if the controller's internal data is synchronized with the grid and DER operating frequencies. An event in the Connect state will make the DER Switch to transition to Disconnect state. An event in the Disconnected state will prevent the operation of synchronization function.

The DER Switch controller provides both instantaneous and time over current relay functions. Additional neutral and ground time overcurrent relay functions are also implemented. The neutral current relay function can be used for 4 wire applications. The trip threshold levels and time delay before tripping are independently adjustable. The calculations for this algorithm are performed when in Connect state. Any triggered event makes the controller transition to the Disconnect state.

The DER Switch controller evaluates single and three phase power flow at the switch and at a remote location. Remote measurement is possible if additional CTs are wired into the DER Switch controller. Independent thresholds are available for switch and remote reverse power. The power measurement is compared with an adjustable threshold and time delay for coordination. If the power flow crosses the threshold then an event is set. The user can select if the crossing occurs in the positive or negative direction for the event to occur. An event in the Connect state will cause the DER Switch to transition to the Disconnect state.

The IEEE 1547 standard contains control requirements that should be satisfied for the interconnection of DER to the grid [5]. Other standards such as UL1741 and State standards such as California Rule 21 reflect many of the underlying concerns that are addressed by the IEEE 1547 series standards [6]. The main control functions required for the DER Switch to meet the IEEE 1547 standard relate to: under and over voltage, under and over frequency, harmonics, DC injection, 


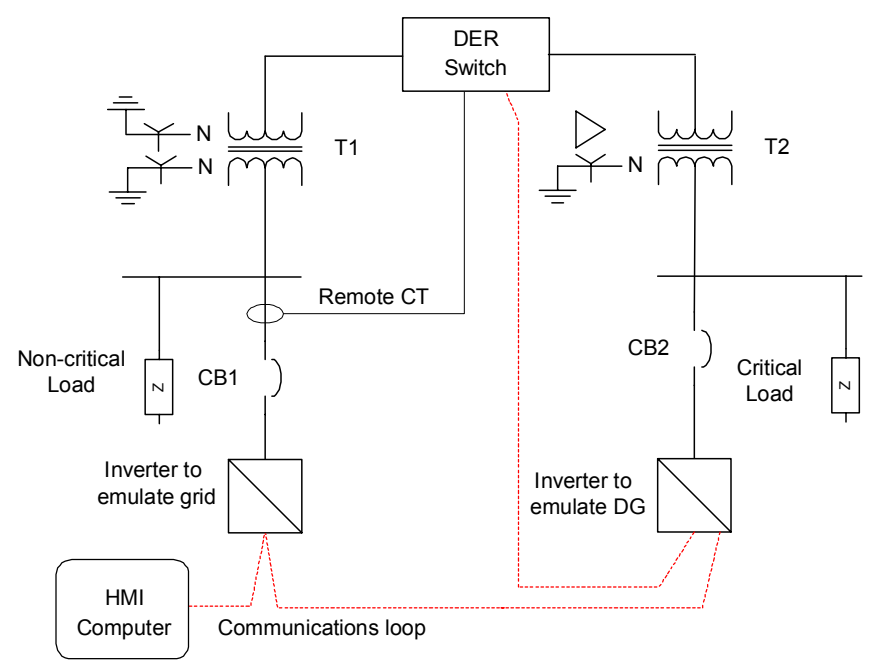

Fig. 5. Setup for testing the DER Switch using inverter to emulate grid and DG along with critical and non-critical loads.

anti-islanding, synchronization, and reconnection timing. The reverse power flow monitored at the point of aggregate loading is used to detect unintentional islanding. The DER Switch is programmed with relay functions to be fully compliant with the IEEE 1547 standard and has the flexibility to meet additional requirements. In addition to the above relay functions it is possible to manually set a digital bit to simulate the occurrence of an event. This was used to verify the proper operation of the DER Switch controller during testing.

\section{RESULTS}

The tests performed included general commissioning followed by the various operational tests. The general commissioning tests were done to ensure the switch's manufacturing integrity. The operational tests concentrated on confirming the operation of the various control algorithms including the prototype's performance, relay functions, IEEE 1547 functions and power quality functions. A representative test power system indicating DER assets, loads, the DER Switch and the area electric power system is shown in Fig. 5. The test facility consists of two power converters, one which is used to emulate the grid and the second which is used to emulate the DG. Transformers and loads in the connection can be switched in to study various grounding options, power flow, and grid conditions.

Fig. 6 shows the connection transition of the circuit breaker. When the switch is not connected, the waveforms at the grid and DG side are distinctive since the DG inverter was not generating a perfect sine wave due to the test setup arrangement. However, the voltage magnitudes in RMS basis and frequency were almost identical and are within the synchronization window. The step signal indicates the command given from the switch controller to the circuit breaker. Scope channels 1 and 2 are the grid and DG voltages, respectively. It was observed that the both voltages would drift and eventually be in phase, which allowed the switch to close. When the switch is connected the voltage seen at the grid and DG outputs are equal and the switch would allow current to flow. The

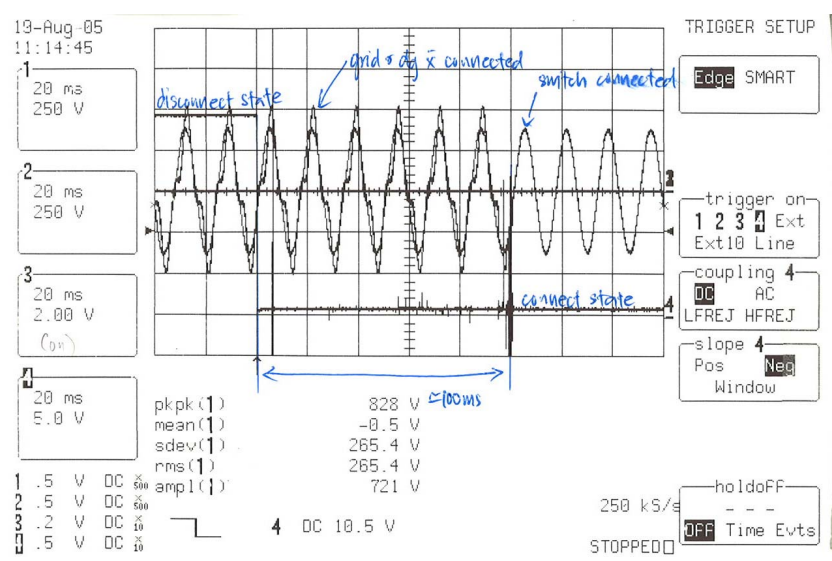

Fig. 6. Grid and DG side voltage and command to circuit breaker waveforms that indicate the DER Switch connection transition.

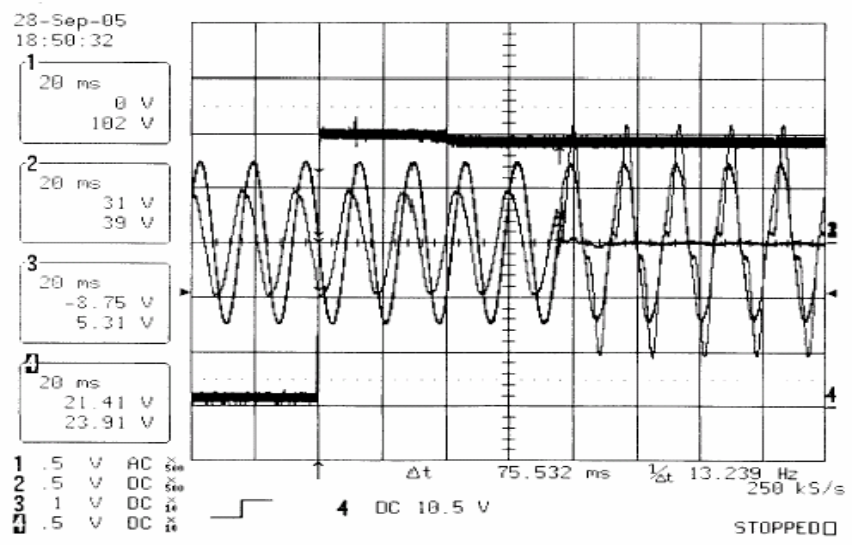

Fig. 7. Grid and DG side voltage, current, and command to circuit breaker waveforms showing switch disconnection transition.

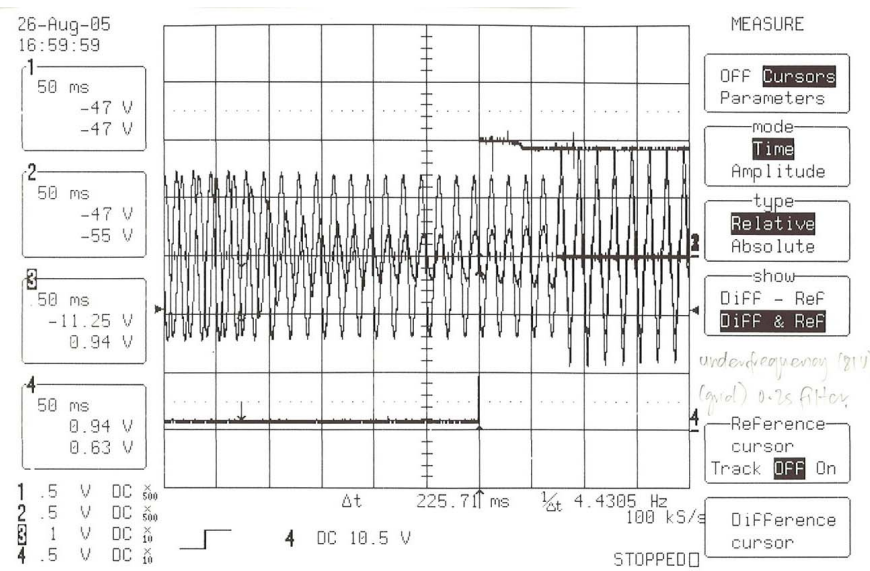

Fig. 8. Response of the system to a frequency event resulting in the DER Switch opening.

switch disconnection transition, which can be seen in Fig. 7, has the similar idea but opposite to the description provided above. The waveforms in Fig. 8 are the grid and DG voltages and current through the switch and the digital command to the circuit breaker. It is observed in the plot that the current waveform ramped down when there was a frequency change of either the grid or the DG inverters. The tripping of the switch occurred after a delay corresponding to the frequency relay coordination settings. 


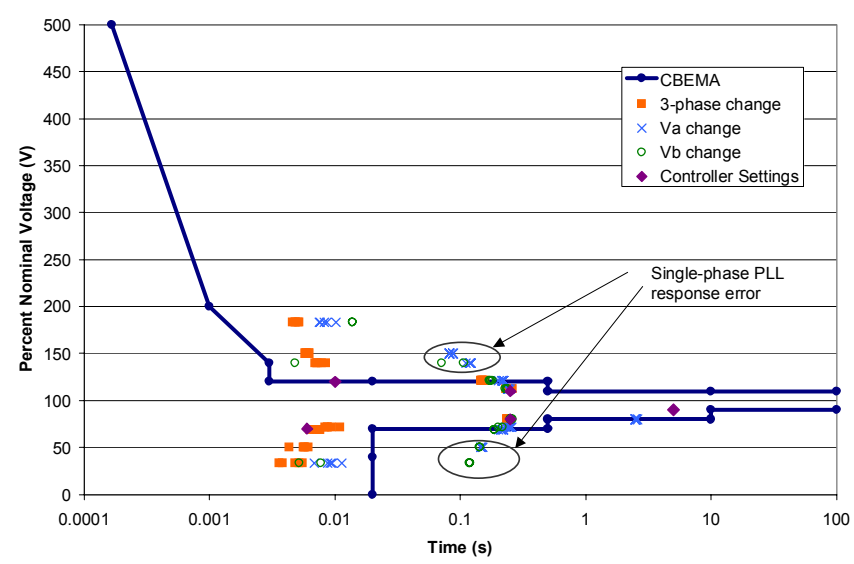

Fig. 9. CBEMA curve requirements, the DSP controller settings and test results performed on three phase and single phase basis.

The DER Switch had the capability of rapidly disconnecting the grid and DG terminals from each other if the switch senses any power quality disturbance according to the ITIC/CBEMA curve requirements. A few tests were performed to verify that the switch responds as expected, which is to disconnect the grid and DG terminals, when a CBEMA power quality event occurs. The magnitude and time response were also confirmed to meet the specified values. The power quality function in the switch controller is represented by 5 magnitude and time response settings that can be adjusted by the user via the HMI. Actual values that approximate the CBEMA curve based on the DSP controller voltage and timer settings were tested. From the initial observation, the switch managed to respond to the specified interruption voltage within the specified time setting. The time response, however, was limited by the circuit breaker disconnection time. A graph of the CBEMA curve with the test data overlaid is shown below in Fig. 9. The results obtained include the 3-phase and single-phase responses. The blue line represents the CBEMA curve, purple dots represents the actual magnitude and time settings specified in the Engineering HMI and the three other marker types indicate the response of the switch to the magnitude change. The majority of the test points responded within the vicinity of the specified magnitude and time settings. However, there are a few responses, as noted by the ovals on the plot, which occurred with a longer transition time than expected. Additional simulations were done to investigate this behavior. Response to very fast sub-cycle, single-phase voltage fluctuation is a very demanding requirement. Synchronous reference frame transformation used to generate phase information from the phase lock loop (PLL) algorithm caused the reaction delay especially when dealing with unbalanced 3phase voltages. It was observed that the PLL responded cleanly to the three-phase voltage test, permitting the fastest reactions designed into the algorithm. The breaker opening delay, a mechanical limitation, prevented the unit from optimally complying with the CBEMA curve. On average, the breaker would fully disconnect no faster than $90 \mathrm{~ms}$ after the most severe transients. It was seen from the test result that a three phase response setting can be programmed to meet the CBEMA voltage curve characteristics. However, the same setting can be less sensitive for the single phase CBEMA event. Detailed results of all the tests are published in [7].

\section{CONCLUSIONS}

The test results resulted in successful meeting of the IEEE 1547 requirements. The complete control of the DER Switch is implemented in a single DSP package. The DSP activates switch operation, performs protective relaying functions and is compatible with the HMI software for enterprise energy management. The integration of all these functions into a single DSP helps achieve the overall equipment reduction target. The use of standard commercially available components for the design and use of components well within the ratings margin helps achieve the reliability goals for the switch. More detailed tests and analysis would be required to obtain the exact reliability characteristics of the DER Switch. The decoupling of the interconnection switch from the DG equipment allows for fast repair with the only limitation being that of spares availability. The ability to identify unintentional islanding situations in a fast and reliable way and to resynchronize was demonstrated during the test program. The present HMI implemented in the DER Switch is an engineering interface that provided full flexibility in making changes to settings. This also allows easy testing and data collection from the prototype DER Switch.

\section{ACKNOWLEDGMENT}

The authors gratefully acknowledge the contributions of $\mathrm{J}$. Lynch, I. Vihinen, B. Freeman, K. Kumar, and G. Kreis for their help at various stages of the DER Switch project.

\section{REFERENCES}

[1] F.Z. Peng, "Editorial - Special issues on Distributed generation," IEEE Transactions on Power Electronics, Vol. 19, No 5, pp. 1157-1158, Sept. 2004.

[2] Information Technology Industry Council, CBEMA/ITIC curve. Available: http://www.itic.org/archives/iticurv.pdf

[3] Robert Lasseter, Abbas Akhil, Chris Marnay, John Stephens, Jeff Dagle, Ross Guttromson, A. Sakis Meliopoulous, Robert Yinger, and Joe Eto, "Integration of distributed energy resources. The CERTS Microgrid Concept" (April 1, 2002). Lawrence Berkeley National Laboratory. Paper LBNL-50829. Available: http://repositories.cdlib.org/lbnl/LBNL50829

[4] Consolidated Edison company of New York, "Specification EO-2115: Handbook of General Requirements for Electrical Service to Dispersed Generation Customers," 2005.

[5] IEEE Standard for Interconnecting Distributed Resources with Electric Power Systems, IEEE Std. 1547 - 2003, June 2003.

[6] US Department of Energy, Distributed Energy Program, Interconnection standards development. Available: http://www.eere.energy.gov/de/interconnection_stan_dev.html

[7] J. Lynch, V. John, S.M. Danial, E. Benedict, I. Vihinen, B. Kroposki and C. Pink, "Flexible DER Utility Interface System, Final report," NREL/TP-560-39876, Aug. $2006 . \quad$ Available: http://www.nrel.gov/docs/fy06osti/39876.pdf 